\title{
Production, characterization and immunogenicity of $P$ particles derived from norovirus GII.4 genotype 2004 variant
}

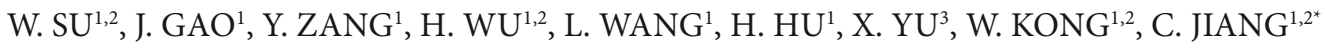

\begin{abstract}
${ }^{1}$ National Engineering Laboratory for AIDS Vaccine, School of Life Science, Jilin University, Changchun 130012, P. R. China; ${ }^{2}$ Key Laboratory for Molecular Enzymology and Engineering, the Ministry of Education, School of Life Science, Jilin University, Changchun 130012, P. R. China; ${ }^{3}$ Biology Group, High School Attached to Northeast Normal University, Changchun 130000, P. R. China
\end{abstract}

Received August 26, 2014; accepted February 9, 2015

\begin{abstract}
Summary. - Norovirus (NoV) is the main cause of nonbacterial infectious gastroenteritis. Due to the difficulty of culturing the virus, research on vaccine against $\mathrm{NoV}$ is focused on virus-like particles (VLPs). On the other hand, the $\mathrm{P}$ particles assembled from the $\mathrm{P}$ domains of $\mathrm{NoV}$ capsid protein become a promising vaccine candidate. GII.4 is the most prevalent genotype of NoV. While the immunogenicity of P particles derived from the GII.4 1996 variant has been investigated, the research on P particles of more recently prevalent variants is lacking. In this study, the P domain of the capsid protein of GII.4 genotype 2004 variant was expressed in Escherichia coli, purified and auto-assembled into P particles of 14-25 nm. Immunization with P particles induced specific serum antibodies with titers of 245,600 and 145,700 in mice and rabbits, respectively. The GII.4 NoV 2004 variant bound to type A, B and O secretor-positive saliva and immune sera blocked this binding, suggesting induction of neutralizing activity in such sera. Thus, this study demonstrated the immunogenicity of NoV P particles generated from E. coli and provided evidence supporting the development of this approach.
\end{abstract}

Keywords: Norovirus; P particle; histo-blood group antigens

\section{Introduction}

Norovirus (NoV) has prevailed as a common pathogen of human gastroenteritis for decades. Since the 1960s, NoV infection has caused numerous outbreaks throughout the world (Bitler et al., 2013). Despite the fact that most clinical symptoms of NoV infections are mild and self-limiting, such as diarrhea, vomiting and fever, severe cases, which result in death, appear occasionally (Frenck et al., 2012;

*Corresponding author. E-mail: jiangcl@jlu.edu.cn; phone: 860431-85167674. Postal address: National Engineering Laboratory for AIDS Vaccine, School of Life Science, Jilin University, Qianjin Street No. 2699, Changchun 130012, P. R. China.

Abbreviations: $\mathrm{HBGAs}=$ histo-blood group antigens; $\mathrm{NoV}(\mathrm{s})=$ Norovirus(es); TEM = transmission electron microscopy; VLPs = virus-like particles
Trivedi et al., 2013). NoVs are genetically classified into five genogroups (GI-GV), of which GII.4 genotype is the cause of most epidemics among humans in the last 20 years (Zheng et al., 2006). Since the 1990s, GII.4 NoV has evolved continuously under selective pressure and differentiated into various genetic variants with altered binding profiles to ligands (human histo-blood group antigens, HBGAs) to escape host immunity (Siebenga et al., 2010).

As a member of the Caliciviridae family, NoV has a 7.5 $\mathrm{kb}$ genome comprising three ORFs (Hardy and Estes, 1996). The capsid of NoV is composed of 180 copies of the major capsid protein VP1, which is the product of ORF2 (Morillo and Timenetsky Mdo, 2011). A single VP1 capsid protein can be divided to two structural domains, the $\mathrm{S}$ (shell) domain and the $\mathrm{P}$ (protrusion) domain (Morillo and Timenetsky Mdo, 2011). To date, no vaccine has been approved to prevent NoV infection. Previous NoV vaccine 
studies were commonly based on virus-like particles (VLPs) (Jiang et al., 1992). VLPs produced from the baculovirusinsect cell expression system have been demonstrated to be immunogenic and provide protection in animal and clinical experiments and are considered as a candidate NoV vaccine (Herbst-Kralovetz et al., 2010; Atmar et al., 2011). Tan et al., however, established a novel P particle vaccine assembled from the VP1 P domain protein (Tan et al., 2008; Tan and Jiang, 2012). NoV P particles are auto-assembled into centrosymmetric particles composed of 24-mer P domain proteins (Tan and Jiang, 2005; Tan et al., 2008). Immunization with P particles has been shown to induce considerable levels of antigen-specific serum antibodies in mice, which could block NoV VLP binding to HBGA ligands, suggesting neutralizing activity (Tan et al., 2008). P particles derived from the GII.4 1996 variant (VA98387/1998 strain) could induce equivalent levels of serum antibodies that blocked the VLP-ligand binding, and of cellular immunity compared with those of VLPs (Tan et al., 2008; Fang et al., 2013). However, levels of antibodies and cellular immunity induced by P particles of another GII.4 1996 variant (95M-14/1995 strain) were weaker than those induced by VLPs (Koho et al., 2012; Tamminen et al., 2012).

The studies mentioned above indicated that the immunogenicity of NoV P particles can be variable and perhaps dependent on the virus strain. Nevertheless, the ease of $\mathrm{NoV}$ $\mathrm{P}$ particle production systems support their development as candidate vaccines. Prior P particle studies focused on GII.4 variants, which are no longer prevalent. The Hunter strain of 2004, one of the latter NoV GII.4 variants that has caused sharply increased numbers of reported $\mathrm{NoV}$ outbreaks throughout the world (Siebenga et al., 2009), as well as maintained prevalence in East Asia and Europe from 2004 to 2006 (Bull et al., 2006; Cheng et al., 2010; Phan et al., 2007), may be an appropriate resource for examining immunogenicity of $\mathrm{P}$ particles. In this study, $\mathrm{P}$ particles of the $\mathrm{NoV}$ GII.4 2004 variant (Hunter504D/04O strain) were produced, purified and characterized. Purified P particles were used for immunization of mice and rabbits to assess their ability to induce NoV-specific antibodies and neutralizing activity in the serum and to bind to HBGAs in saliva.

\section{Materials and Methods}

Saliva samples. A total of 22 saliva samples were collected from 22 healthy adult Chinese volunteers who gave informed consent to participate in this study. The saliva samples were boiled at $100^{\circ} \mathrm{C}$ immediately after collection and centrifuged at $10,000 \times g$ for $10 \mathrm{~min}$. The supernatant was stored at $-20^{\circ} \mathrm{C}$ until use. The secretor-positive HBGA types of the saliva samples were determined using Blood Grouping Reagents (Brother Biotech, Changchun, China).
Expression and purification of P domain protein. The GII. $4 \mathrm{NoV}$ 2004 variant (Hunter504D/04O strain, GenBank DQ078814) P domain coding sequence DNA ( $948 \mathrm{bp}$ ) followed by a C-terminal Histag was synthesized by Generay Biotech Co., Ltd. (Shanghai, China), and cloned in the pET-28a $(+)$ prokaryotic expression vector (Novagen, Darmstadt, Germany) using NcoI and XhoI restriction sites. The recombinant construct was transfected into Escherichia coli (BL21), and the expression of $\mathrm{P}$ domain gene was induced with $0.25 \mathrm{mmol} / \mathrm{l} \mathrm{IPTG}$ at $16^{\circ} \mathrm{C}$ overnight as described previously (Tan et al., 2011). Purification of the recombinant His-tag proteins was carried out using Ni affinity chromatography (GE Healthcare, Piscataway, NJ, USA) at the eluting imidazole concentrations of $250 \mathrm{mmol} / \mathrm{l}$ and $500 \mathrm{mmol} / \mathrm{l}$.

Gel filtration chromatography. Gel filtration chromatography with the Akta fast performance liquid chromatography (FPLC) system (GE Healthcare) was described previously (Tan et al., 2011). Briefly, proteins were loaded on a Superdex 200 column (Amersham Bioscience, Piscataway, NJ, USA), separated by molecular weight and collected as fractions. Gel filtration calibration kits (GE Healthcare) were used to calibrate the molecular weight of the eluted fractions.

PAGE. For PAGE, proteins were dissolved in the native loading buffer ( $0.5 \mathrm{~mol} / \mathrm{l} \mathrm{pH} 6.8$ Tris-HCl, $50 \%$ glycerol, $0.1 \%$ bromophenol blue) and analyzed by electrophoresis in a $10 \%$ native gel without SDS, followed by staining with Coomassie Blue. For SDS-PAGE, proteins were dissolved in the denaturing loading buffer $(0.5 \mathrm{~mol} / \mathrm{l}$ pH 6.8 Tris- $\mathrm{HCl}, 50 \%$ glycerol, $10 \%$ SDS, $5 \% \beta$-mercaptoethanol, $0.1 \%$ bromophenol blue) and analyzed by electrophoresis in a $10 \%$ SDS gel, followed by staining with Coomassie Blue.

Western blot analysis. Unstained proteins separated in the SDS-PAGE gel were blotted to a PVDF membrane. After blocking with $5 \%$ nonfat milk and washing in Tris-buffered saline-Tween solution, the blot was incubated with a primary mouse anti-His tag monoclonal antibody (1:200 dilution, Sigma, St. Louis, MO, USA) for $1 \mathrm{hr}$, washed and then incubated with a secondary goat antimouse IgG antibody conjugated to alkaline phosphatase (1:5,000 dilution, Sigma) and detected using an enhanced chemoluminescence kit (Sigma).

Dynamic light scattering. Sample solutions were passed through $0.45 \mu \mathrm{m}$ filters and equilibrated at room temperature for $10 \mathrm{~min}$ prior to measuring. Size distributions of $\mathrm{P}$ particles were obtained by dynamic light scattering using a Zetasizer NANO ZS90 instrument (Malvern, Worcestershire, UK) at a fixed scattering angle of $90^{\circ}$ and at $25^{\circ} \mathrm{C}$.

Transmission electron microscopy (TEM). P particle solutions were mixed with an equal amount of $1 \%$ phosphotungstic acid (PTA, adjusted with $\mathrm{NaOH}$ to $\mathrm{pH}$ 6.5). The mixed solution was placed onto a carbon support film on a copper grid for $5 \mathrm{~min}$. After wicking away excess solution, the grids were dried in the vacuum for $5 \mathrm{~min}$. The samples were viewed with a H-7650 TEM (Hitachi, Tokyo, Japan) at an $80 \mathrm{kV}$ accelerating voltage.

Immunization. Six-week-old female BALB/c mice and threeweek-old New Zealand white rabbits were immunized in this 
study. The permissions of animal experiment are SYXK (JL) 2013-0002 for mice and SYXK (JL) 2012-0012 for rabbits, approved by the Committee on Animal Research of Jilin University. For mice, each group $(n=6)$ was immunized three times at 2 -week intervals by subcutaneous injection of $10 \mu \mathrm{g}$ of $\mathrm{P}$ particles (in $60 \mu \mathrm{PBS}$ ) on the back. Each group of rabbits $(\mathrm{n}=2)$ was immunized three times at 1 -week intervals by injection of $400 \mu \mathrm{g}$ of P particles (in $1 \mathrm{ml}$ PBS) subcutaneously at multiple sites on the back. As a control for both species of animals, PBS of the equivalent volume was injected. Freund's adjuvant was given to enhance the immune response in both mice and rabbits (Freund's complete adjuvant in $1^{\text {st }}$ injection, Freund's incomplete adjuvant in the other two injections). Blood was collected from mice 2 weeks after each immunization and from rabbits 1 week after each immunization.

Titration of antibodies. An ELISA was used to measure immune reactivity and antibody titers of mouse and rabbit antisera after immunization with $\mathrm{P}$ particles. Ninety-six-well microtiter plates were coated with $0.1 \mu \mathrm{g}$ of $\mathrm{P}$ particle as the antigen at $4^{\circ} \mathrm{C}$ overnight, blocked with $5 \%$ nonfat milk and then incubated with immunized animal sera at indicated dilutions for $1 \mathrm{hr}$ at $37^{\circ} \mathrm{C}$. Subsequently, the plates were incubated with the secondary HRP-conjugated goat anti-mouse/rabbit IgG antibody (diluted 1:5,000, Sigma) for $1 \mathrm{hr}$ at $37^{\circ} \mathrm{C}$ and then detected using a TMB Liquid Substrate System (Sigma). The P particle-bound antibody titer was defined as the end-point dilution with the cut-off signal intensity of 0.15 .

ELISA of P particle binding to saliva. To test the $\mathrm{P}$ particle binding to saliva samples by ELISA, microtiter plates were coated with type A, B and $\mathrm{O}$ saliva samples (1:100 dilution) at $4^{\circ} \mathrm{C}$ overnight. After blocking with $5 \%$ nonfat milk, P particles $(0.1 \mu \mathrm{g}$ in $100 \mu \mathrm{l}$ PBS $)$ were added, followed by an incubation for $1 \mathrm{hr}$ at $37^{\circ} \mathrm{C}$. Thereafter, the plates were incubated with antiserum from rabbits immunized with $\mathrm{P}$ particles at a dilution of $1: 300$ for $1 \mathrm{hr}$ at $37^{\circ} \mathrm{C}$, followed by an incubation with a secondary HRP-conjugated goat anti-rabbit IgG antibody (1:5,000 dilution, Sigma, St. Louis, MO, USA) for $1 \mathrm{hr}$ at $37^{\circ} \mathrm{C}$. Signal was detected with a TMB Liquid Substrate System (Sigma). The experiment was repeated twice.

Competitive ELISA of blocking of the P particle-binding to saliva by immune antisera. To test the ability of animal anti-P particle antisera to block the binding of $\mathrm{P}$ particles to saliva, a competitive ELISA was used. Briefly, microtiter plates were coated with type $\mathrm{A}, \mathrm{B}$ and $\mathrm{O}$ saliva samples (1:100 dilution) at $4^{\circ} \mathrm{C}$ overnight, followed by blocking with $5 \%$ nonfat milk. P particles $(0.1 \mu \mathrm{g}$ in $100 \mu \mathrm{l} \mathrm{PBS})$ were pre-incubated with serially diluted anti-P particle mouse serum for $30 \mathrm{~min}$ at $37^{\circ} \mathrm{C}$. The pre-incubated mixtures were added to the plates, incubated for $1 \mathrm{hr}$ at $37^{\circ} \mathrm{C}$, and then the unbound material was carefully washed away. The plates were further incubated with immunized rabbit serum at the dilution of 1:300 for $1 \mathrm{hr}$ at $37^{\circ} \mathrm{C}$, followed by a secondary HRP-conjugated goat anti-rabbit IgG antibody (1:5,000 dilution, Sigma) at $37^{\circ} \mathrm{C}$ for $1 \mathrm{hr}$. Signal was detected with the TMB Liquid Substrate System (Sigma). The experiment was repeated twice.
Statistical analysis. Statistical analysis was performed with SPSS 17.0 (SPSS, Chicago, IL, USA) using the two-tailed Student's $t$ test. Data are expressed as the mean \pm SD.

\section{Results}

\section{Production of $P$ domain protein and $P$ particles}

The study was initiated by cloning the GII.4 2004 variant (Hunter504D/04O) NoV P domain coding sequence with a C-terminal His-tag into the pET-28a $(+)$ prokaryotic expression vector (Fig. 1a). The plasmid was transformed into E. coli, and after IPTG induction an approximately 35 $\mathrm{kD}$ protein was detected in the supernatant by SDS-PAGE (Fig. 1b). We subsequently purified the $35 \mathrm{kD} \mathrm{NoV} \mathrm{P} \mathrm{do-}$ main protein by $\mathrm{Ni}$ affinity chromatography (Fig. 1c) and confirmed its presence by Western blot analysis using a His antibody (Fig. 1d).

\section{Characterization of $P$ particles}

Several approaches were utilized in this study to determine the assembly characteristics of $\mathrm{P}$ domain proteins. PAGE analysis of the $\mathrm{P}$ domain proteins showed several bands much larger

(a)

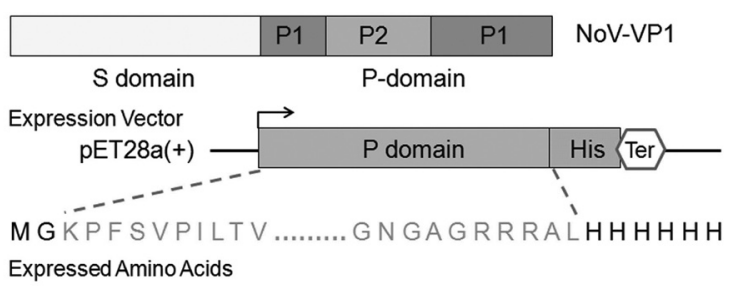

(b)

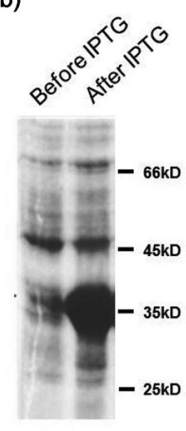

(c)

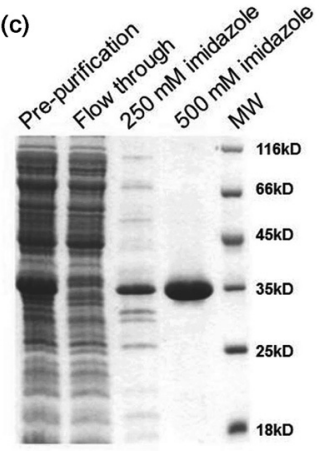

(d)

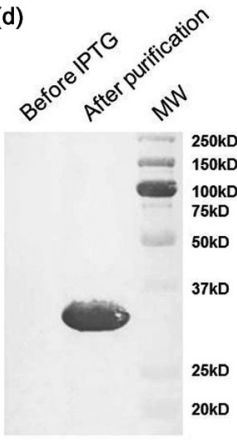

Fig. 1

\section{Production of NoV $P$ domain protein}

(a) Vector expressing His-tagged P domain protein, (b) SDS-PAGE, expression of $\mathrm{P}$ domain protein in E. coli after IPTG induction, (c) SDS-PAGE, $\mathrm{Ni}$ affinity chromatography of $\mathrm{P}$ domain protein eluted with imidazole, (d) Western blot analysis of $\mathrm{P}$ domain protein. 


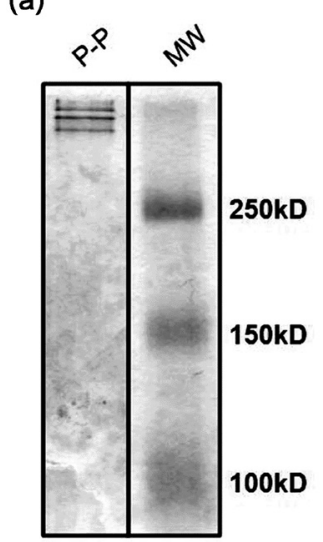

(b)

(c)

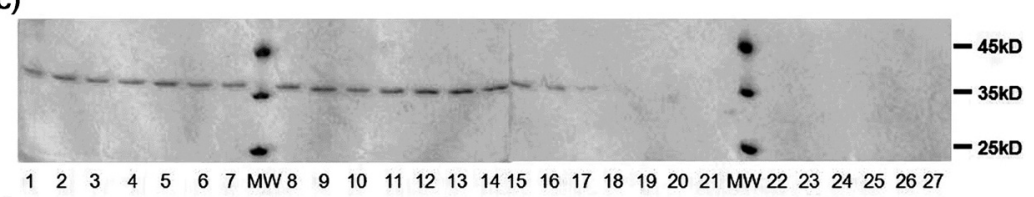

(d)

Fig. 2

Characterization of NoV $P$ particles (a) native PAGE of P particles, (b) gel filtration chromatography of $\mathrm{P}$ particles purified by $\mathrm{Ni}$-affinity chromatography, $\mathrm{mAU}=$ milli-absorbance unit, (c) SDS-PAGE of eluted fractions 1-27 from (b), (d) dynamic light scattering analysis of $P$ particles from the peak fraction in (b), (e) TEM of $\mathrm{P}$ particles from the peak fraction in (b), scale bar $=100 \mathrm{~nm}$.

Fig. 3

Induction of serum antibodies in mice and rabbits by $P$ particles

(a, c) ELISA of antibodies after immunizations 1-3, (b, d) Antibody titers of sera after the 3rd immunization. $\mathrm{P}-\mathrm{P}=\mathrm{P}$ particle. ${ }^{* *}: \mathrm{P}<0.01$, compared with control.
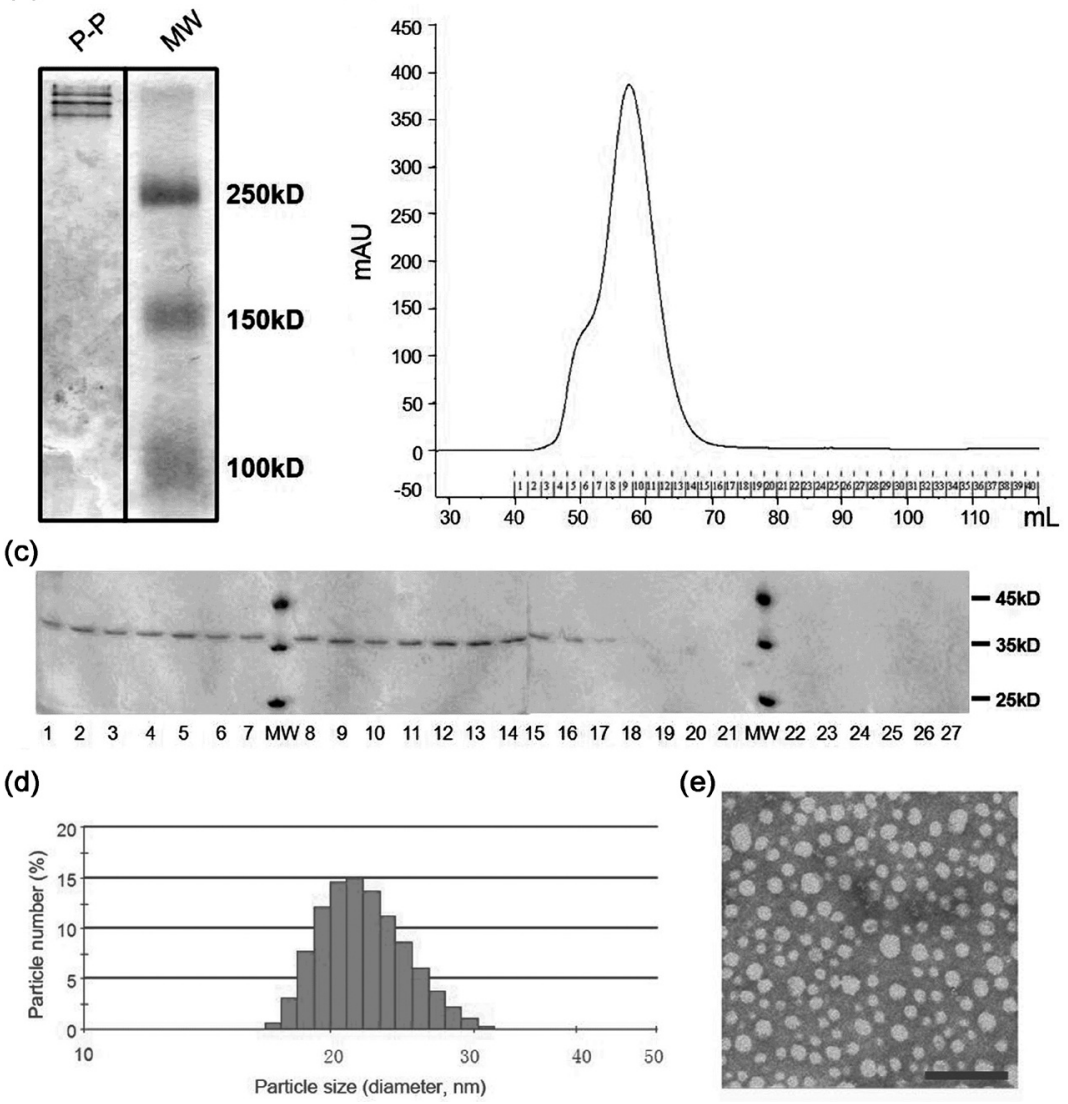

(e) (a)

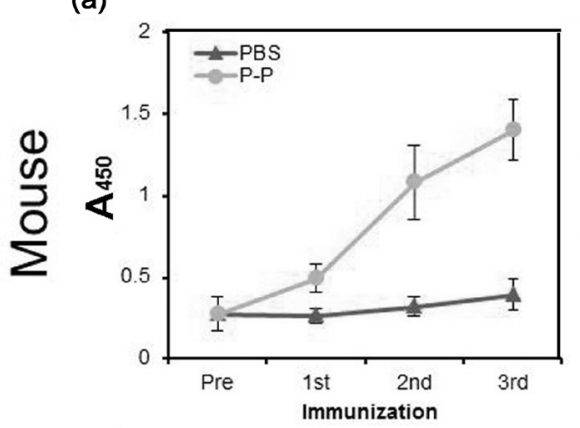

(c)

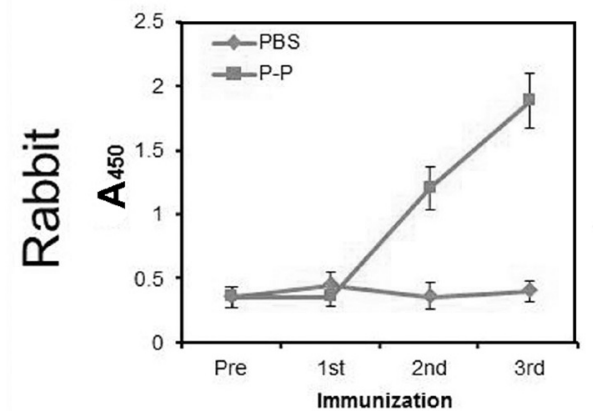

(b)

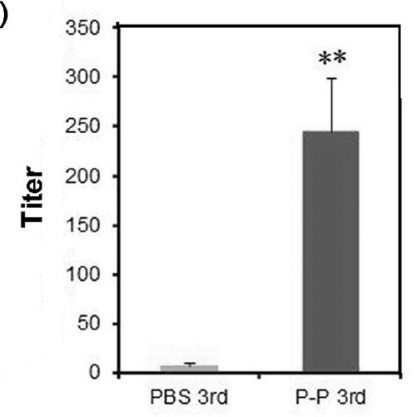

(d)

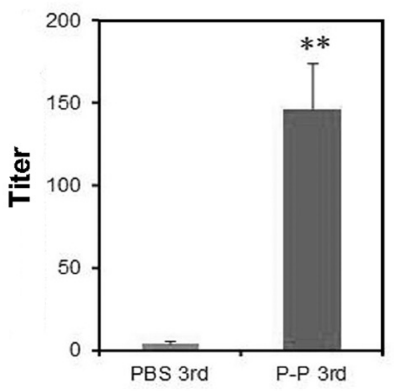


than $250 \mathrm{kD}$ (Fig. 2a), suggesting that they automatically assembled into the advanced $\mathrm{P}$ particle structure. The gel filtration chromatography curve for the $\mathrm{P}$ particles exhibited a peak at $\sim 840 \mathrm{kDa}$ (Fig. 2b). Fractions including the peak were collected, and the composition was analyzed by SDS-PAGE (Fig. 2c). Dynamic light scattering measurements indicated the size (diameter) of $\mathrm{P}$ particles to be $\sim 22 \mathrm{~nm}$ and within the range of 17-30 nm (Fig. 2d). Electron microscopy also revealed globular particles with diameters of 14-25 nm (Fig. 2e), indicating that GII.4 2004 variant NoV P particles were produced and could be used in subsequent immunization procedures.

\section{Induction of specific antibodies by $P$ particles}

Mice and rabbits were immunized subcutaneously three times with NoV P particles. After each immunization, sera were collected, and antibodies against $\mathrm{P}$ particles were detected. In both mice and rabbits, $\mathrm{P}$ particles induced antibody immune responses. Detection by ELISA showed that the generation of specific antibodies was enhanced increasingly following each subsequent immunization, and just two immunizations were sufficient to induce significant levels of anti-NoV $\mathrm{P}$ particle antibodies in both mice and rabbits (Fig. 3a and 3c). Further examination revealed antibody titers of 245,600 in mouse sera and 145,700 in rabbit sera after three immunizations (Fig. $3 \mathrm{~b}$ and $3 \mathrm{~d}$ ).

\section{Binding of $P$ particles to saliva of type $A, B$ and $O$}

To identify the ligands for GII.4 2004 variant NoV, binding assays of secretor-positive saliva samples of type A, B and $\mathrm{O}$ to $\mathrm{P}$ particles were performed. The results demonstrated that all three types of saliva samples could bind $\mathrm{P}$ particles. Furthermore, the affinity rank from the highest to the lowest was type A, type B and type O, respectively (Fig. 4).

\section{Inhibition of $P$ particle-binding to saliva by immune sera}

As there are currently no cell or animal models for norovirus infection available and we are unable to culture live human $\mathrm{NoV}$, the neutralization activity of antisera is represented by their ability to block NoV sub-viral particle-binding to HBGA ligands (Tan et al., 2008; Tamminen et al., 2012). In this study, we performed the blocking assays to measure the inhibition of $\mathrm{P}$ particle-binding to saliva by mouse antisera. As a result, antibodies in mouse sera blocked $\mathrm{P}$ particle-binding to saliva samples in a dose-dependent manner (Fig. 5), suggesting that the immunization with $\mathrm{P}$ particles induced antibodies with neutralization activity. Neutralization activity was higher against $\mathrm{P}$ particle-binding to type A and B saliva samples, whereas it was lower when compared with that to type O (Fig. 5), implying different modes of blocking of these interactions by the serum antibodies.

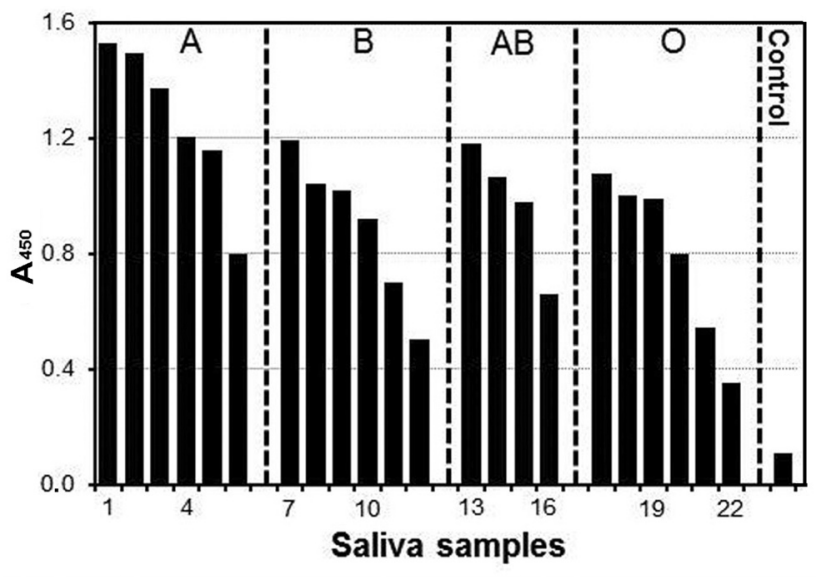

Fig. 4

Binding of $P$ particles to saliva samples of type A, B and O (ELISA)
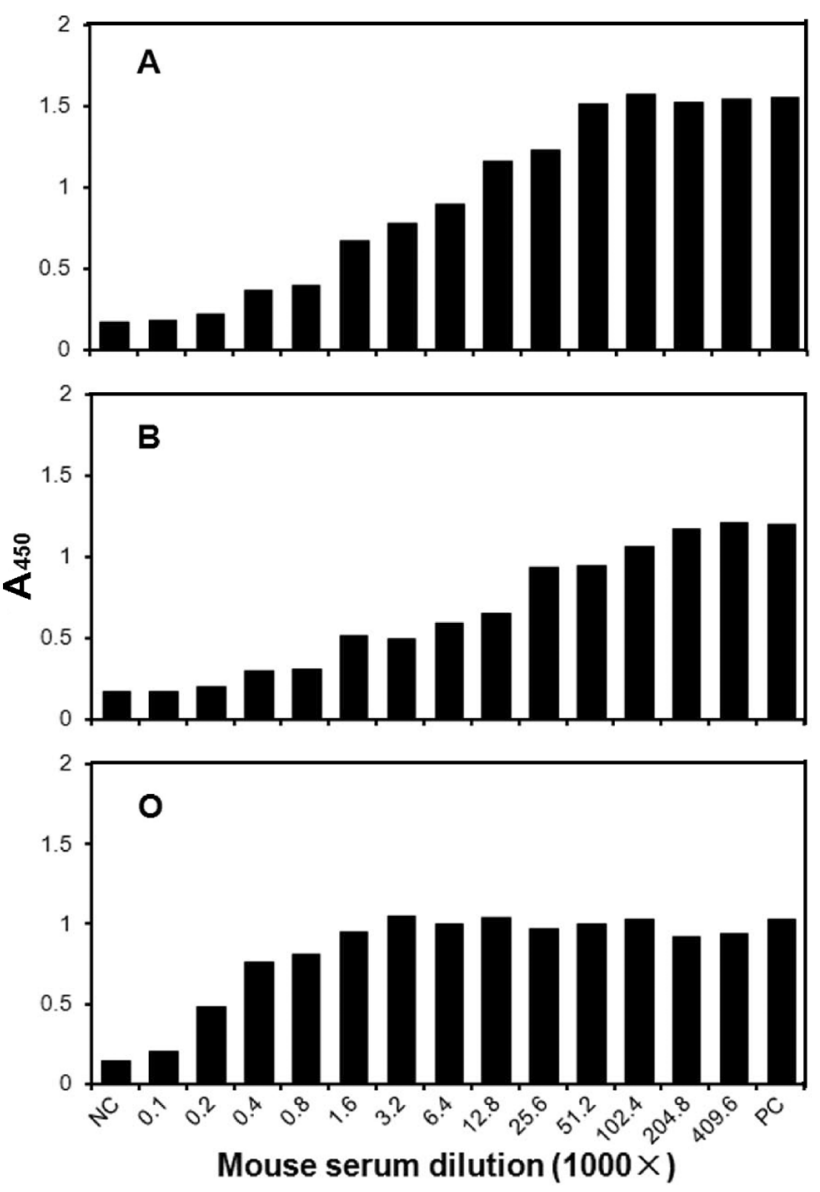

Fig. 5

Blocking of $P$ particle-binding to saliva samples by immune sera of type $A, B$ and $O$

ELISA. NC: P particles were replaced by PBS. PC: mouse anti-sera were replaced by $\mathrm{PBS}$. 


\section{Discussion}

The physiological characteristics and immunogenicity of $P$ particles, as a novel vaccine candidate for NoV, have been investigated previously (Tan et al., 2008; Tan and Jiang, 2012). Although P particles derived from the GII.4 1996 variant (VA98387/1998) were demonstrated to exhibit considerable immunogenicity, this strain has not been prevalent for a decade (Tan et al., 2008). Tamminen et al. reported that the antiserum raised against P particles of another GII.4 1996 variant (95M-14/1995) could bind only to the same NoV strain but not to other GII.4 strains or genotypes, suggesting the lack of cross-reactivity (Tamminen et al., 2012). To investigate $\mathrm{P}$ particles from a more recently prevalent NoV, the GII.4 2004 variant (Hunter504D/04O) was chosen for the characterization in terms of production and immunogenicity. This strain, which caused outbreaks throughout the world after 2004 is $91 \%$ similar to the VA98387/1998 strain and $92 \%$ to the $95 \mathrm{M}-14 / 1995$ strain (Bull et al., 2006; Cheng et al., 2010). The P domain protein of the GII.4 2004 variant $\mathrm{NoV}$ was efficiently expressed in E. coli, eluted to a high extent in the lysate supernatant, and effectively purified by Ni affinity chromatography. Based on the results of various analyses (PAGE, gel filtration chromatography, dynamic light scattering and electron microscopy), $\mathrm{P}$ domain proteins were observed to auto-assemble dynamically into $P$ particles of diverse sizes, with diameters ranging between 14-25 nm (Fig. 2e). A similar assembly profile was described in a previous report on P particles of the VA98387/1998 strain as a mixture of 12-, 18-, 24- and 36-mers (Bereszczak et al., 2012).

Prior studies demonstrated that HBGAs, encoded by the $\mathrm{ABO}$, non-secretor and Lewis gene families, serve as NoV ligands (Huang et al., 2003, 2005, Choi et al., 2008; de Rougemont et al., 2011). In this study, type A, B and $\mathrm{O}$ secretor-positive antigens efficiently bound to the Hunter504D/04O strain, consistent with a previous report (de Rougemont et al., 2011). However, our results determined that the affinity of GII.4 2004 variant NoV P particles to the type A antigen was higher than the affinity to the antigen type $\mathrm{B}, \mathrm{AB}$ and $\mathrm{O}$, suggesting the preference of the Hunter504D/04O strain for binding to the type A antigen. Further characterization by immunizing mice and rabbits with these $P$ particles resulted in the production of considerable serum antibody titers $(245,600$ in mouse sera and 145,700 in rabbit sera) against NoV after the third inoculation. As demonstrated by the competitive ELISA blocking assay, the immune serum antibodies blocked the binding between NoV P particles and saliva samples, suggesting that the $\mathrm{P}$ particles were able to induce neutralizing antibodies. Results of the blocking assay also indicated stronger neutralizing activity of the anti-sera against $\mathrm{P}$ particle binding to type A and type B antigens (with titers of 51,200 and 204,800, respectively) than that to type $\mathrm{O}$ antigens (titer 1600). These results suggest that type $A$ and type $B$ antigen binding sites of the Hunter504D/04O P domain were more sensitive to neutralizing antibodies induced by the $\mathrm{P}$ particle vaccine, implying that it would provide more effective protection for individuals carrying type A and type B antigens.

In conclusion, P particles derived from GII.4 2004 variant NoV were convenient to produce and purify. Importantly, they could induce humoral immune responses with potential neutralizing antibody activity. Thus, this study supports the potential of $\mathrm{P}$ particles as a $\mathrm{NoV}$ vaccine candidate.

Acknowledgements. This study was supported by grants from the National "Twelfth Five-Year" Plan for Science \& Technology Support of China (No. 2012ZX10001-009) and the National Natural Science Fund of China (No.31301179).

\section{References}

Atmar RL, Bernstein DI, Harro CD, Al-Ibrahim MS, Chen WH, Ferreira J, Estes MK, Graham DY, Opekun AR, Richardson C, Mendelman PM (2011): Norovirus vaccine against experimental human Norwalk Virus illness. N. Engl. J. Med. 365, 2178-2187. http://dx.doi.org/10.1056/ NEJMoa1101245

Bereszczak JZ, Barbu IM, Tan M, Xia M, Jiang X, van Duijn E, Heck AJ (2012): Structure, stability and dynamics of norovirus $\mathrm{P}$ domain derived protein complexes studied by native mass spectrometry. J. Struct. Biol. 177, 273-282. http:// dx.doi.org/10.1016/j.jsb.2012.01.005

Bitler EJ, Matthews JE, Dickey BW, Eisenberg JN, Leon JS (2013): Norovirus outbreaks: a systematic review of commonly implicated transmission routes and vehicles. Epidemiol. Infect. 141, 1563-1571. http://dx.doi.org/10.1017/ $\underline{\text { S095026881300006X }}$

Bull RA, Tu ET, McIver CJ, Rawlinson WD, White PA (2006): Emergence of a new norovirus genotype II.4 variant associated with global outbreaks of gastroenteritis. J. Clin. Microbiol. 44, 327-333. http://dx.doi.org/10.1128/ JCM.44.2.327-333.2006

Cheng WX, Ye XH, Yang XM, Li YN, Jin M, Jin Y, Duan ZJ (2010): Epidemiological study of human calicivirus infection in children with gastroenteritis in Lanzhou from 2001 to 2007. Arch Virol. 155, 553-555. http://dx.doi. org/10.1007/s00705-010-0592-5

Choi JM, Hutson AM, Estes MK, Prasad BV (2008): Atomic resolution structural characterization of recognition of histoblood group antigens by Norwalk virus. Proc. Natl. Acad. Sci. USA 105, 9175-9180. http://dx.doi.org/10.1073/ pnas. 0803275105

de Rougemont A, Ruvoen-Clouet N, Simon B, Estienney M, ElieCaille C, Aho S, Pothier P, Le Pendu J, Boireau W, Belliot G (2011): Qualitative and quantitative analysis of the 
binding of GII.4 norovirus variants onto human blood group antigens. J. Virol. 85, 4057-4070. http://dx.doi. org/10.1128/JVI.02077-10

Fang H, Tan M, Xia M, Wang L, Jiang X (2013): Norovirus P particle efficiently elicits innate, humoral and cellular immunity. PLoS One 8, e63269. http://dx.doi.org/10.1371/journal. pone.0063269

Frenck R, Bernstein DI, Xia M, Huang P, Zhong W, Parker S, Dickey M, McNeal M, Jiang X (2012): Predicting susceptibility to norovirus GII.4 by use of a challenge model involving humans. J. Infect. Dis. 206, 1386-1393. http://dx.doi. org/10.1093/infdis/jis514

Hardy ME, Estes MK (1996): Completion of the Norwalk virus genome sequence. Virus Genes 12, 287-290. http://dx.doi. org/10.1007/BF00284649

Herbst-Kralovetz M, Mason HS, Chen Q (2010): Norwalk virus-like particles as vaccines. Expert Rev. Vaccines 9, 299-307. http://dx.doi.org/10.1586/erv.09.163

Huang P, Farkas T, Marionneau S, Zhong W, Ruvoen-Clouet N, Morrow AL, Altaye M, Pickering LK, Newburg DS, LePendu J, Jiang X (2003): Noroviruses bind to human ABO, Lewis, and secretor histo-blood group antigens: identification of 4 distinct strain-specific patterns. J. Infect. Dis. 188, 19-31. http://dx.doi.org/10.1086/375742

Huang P, Farkas T, Zhong W, Tan M, Thornton S, Morrow AL, Jiang X (2005): Norovirus and histo-blood group antigens: demonstration of a wide spectrum of strain specificities and classification of two major binding groups among multiple binding patterns. J. Virol. 79, 6714-6722. http:// dx.doi.org/10.1128/JVI.79.11.6714-6722.2005

Jiang X, Wang M, Graham DY, Estes MK (1992): Expression, selfassembly, and antigenicity of the Norwalk virus capsid protein. J. Virol. 66, 6527-6532.

Koho T, Huhti L, Blazevic V, Nurminen K, Butcher SJ, Laurinmaki P, Kalkkinen N, Ronnholm G, Vesikari T, Hytonen VP, Kulomaa MS (2012): Production and characterization of virus-like particles and the P domain protein of GII.4 norovirus. J. Virol. Methods 179, 1-7. http://dx.doi. org/10.1016/j.jviromet.2011.05.009

Morillo SG, Timenetsky Mdo C (2011): Norovirus: an overview. Rev. Assoc. Med. Bras. 57, 453-458. http://dx.doi. org/10.1016/S0104-4230(11)70094-X

Phan TG, Khamrin P, Akiyama M, Yagyu F, Okitsu S, Maneekarn N, Nishio O, Ushijima H (2007): Detection and genetic characterization of norovirus in oysters from China and Japan. Clin. Lab. 53, 405-412.

Siebenga JJ, Lemey P, Kosakovsky Pond SL, Rambaut A, Vennema H, Koopmans M (2010): Phylodynamic reconstruction reveals norovirus GII.4 epidemic expansions and their molecular determinants. PLoS Pathog. 6, e1000884. http://dx.doi.org/10.1371/journal.ppat.1000884

Siebenga JJ, Vennema H, Zheng DP, Vinje J, Lee BE, Pang XL, Ho EC, Lim W, Choudekar A, Broor S, Halperin T, Rasool NB, Hewitt J, Greening GE, Jin M, Duan ZJ, Lucero Y, O'Ryan M, Hoehne M, Schreier E, Ratcliff RM, White PA, Iritani N, Reuter G, Koopmans M (2009): Norovirus illness is a global problem: emergence and spread of norovirus GII.4 variants, 2001-2007. J. Infect. Dis. 200, 802-812. http:// dx.doi.org/10.1086/605127

Tamminen K, Huhti L, Koho T, Lappalainen S, Hytonen VP, Vesikari T, Blazevic V (2012): A comparison of immunogenicity of norovirus GII-4 virus-like particles and P-particles. Immunology 135, 89-99. http://dx.doi.org/10.1111/j.13652567.2011.03516.x

Tan M, Fang P, Chachiyo T, Xia M, Huang P, Fang Z, Jiang W, Jiang X (2008): Noroviral P particle: structure, function and applications in virus-host interaction. Virology 382 , 115-123. http://dx.doi.org/10.1016/j.virol.2008.08.047

Tan M, Huang P, Xia M, Fang PA, Zhong W, McNeal M, Wei C, Jiang W, Jiang X (2011): Norovirus P particle, a novel platform for vaccine development and antibody production. J. Virol. 85, 753-764. http://dx.doi.org/10.1128/JVI.01835-10

Tan M, Jiang X (2005): The $\mathrm{p}$ domain of norovirus capsid protein forms a subviral particle that binds to histo-blood group antigen receptors. J. Virol. 79, 14017-14030. http://dx.doi. org/10.1128/JVI.79.22.14017-14030.2005

Tan M, Jiang X (2012): Norovirus P particle: a subviral nanoparticle for vaccine development against norovirus, rotavirus and influenza virus. Nanomedicine (Lond) 7, 889-897. http:// dx.doi.org/10.2217/nnm.12.62

Trivedi TK, Desai R, Hall AJ, Patel M, Parashar UD, Lopman BA (2013): Clinical characteristics of norovirus-associated deaths: a systematic literature review. Am. J. Infect. Control 41, 654-657. http://dx.doi.org/10.1016/j.ajic.2012.08.002

Zheng DP, Ando T, Fankhauser RL, Beard RS, Glass RI, Monroe SS (2006): Norovirus classification and proposed strain nomenclature. Virology 346, 312-323. http://dx.doi. org/10.1016/j.virol.2005.11.015 